\title{
Research on experimental method of carbon nanotubes space environment effect
}

\author{
Man Li *, Yuming Liu, Naiyuan Cui, Qiang Yu, Yu Li, and Yongtai Zhang \\ Beijing Institute of Spacecraft Environment Engineering, Beijing, China
}

\begin{abstract}
Keywords: carbon nanotubes, atomic oxygen, debris, space environment effect
\end{abstract}

\begin{abstract}
Due to its unique structure and superior performance, carbon nanotubes are proposed to have great potential applications in many fields. Recently, more and more concerns have been focused on the application of carbon nanotubes in space technology. It is believed that carbon nanotubes can have a broad impact on space missions in future with benefits principally in space technology, such as lightweight structure materials, environment protection materials, energy gerneration and storage and nanoelcetronics. However, carbon nanotubes would suffer chemical and physical damage from the space environment when it was used in spacecraft, just like all the other space materials. The environment where spacecrafts operate is extremely intricate, primarily represented by electromagnetic irradiation, charged particle irradiation, high vacuum, cold and hot alternation, atomic oxygen erosion, clash from space debris, and other factors. The mostly effect in space environment is atomic oxygen and debris for grapheme. This paper is about the research on the experimental methods of carbon nanotubes space environment effect with atomic oxygen and space debris as examples, in a bid to propose the experimental scheme of carbon nanotubes space environment effect. It will be great helpful to promote the applications of carbon nanotubes in space technology.
\end{abstract}

\section{Introduction}

Carbon nanotubes have been a focus for scientists around the world with great interest. The recent years have seen it be the most eye-catching thing, particularly in the field of materials science and condensed matter physics. The world attention to the carbon nanotubes is not only attributed to the abundant and novel physics contained in it, showcasing the important value for theoretical research, but also to its unique structure and superior performance that could make it applied in a variety of sectors. As a result, it will become a new area for growth for economic and social progress in the future. Carbon nanotubes feature sound flexibility, stable chemical property, easy to be modified. These characteristics provide

\footnotetext{
* Corresponding author: $\underline{\text { li-man8319@163.com }}$
} 
the carbon nanotubes with uniquely positioned to be applied in the spacial atomic oxygen protective film and antistatic transparent film ${ }^{[1-3]}$.

However, the most striking difference between aerospace products and other articles is the working environment. The environment where spacecrafts operate is extremely intricate, primarily represented by electromagnetic irradiation, charged particle irradiation, high vacuum, cold and hot alternation, atomic oxygen erosion, clash from space debris, and other factors ${ }^{[4-6]}$. When materials used for manufacturing the spacecraft and components in it are influenced by these environmental factors, they will be subject to degradation in performance and even be damaged. This will bring a seriously negative effect on the working conditions, even the safety of the spacecraft, leading to its failure. Analysis of on-orbit abnormality and faults on spacecrafts home and abroad demonstrates that the spacial environment effect serves as one of the main factors for on-orbit faults, being the most frequent factor among all kinds of factors leading to faults. Under incomplete statistics of all countries, approximately more than $50 \%$ of satellite failures are the result of the space environment. The number of faults caused by the deteriorating performance from the interaction between the space environment and material accounts for $40 \%$ of the total ${ }^{[7,8]}$. Considering the analysis above, any aerospace products before being put into use must be subject to the research on the effects of the space environment, in order to analyze how its performance evolves in the space environment and determine its applicability. This process could ensure the spacecraft to run in a stable manner when it is on orbit. This paper is about the research on the experimental method of carbon nanotubes space environment effect with atomic oxygen and space debris as examples, in a bid to propose the experimental scheme of carbon nanotubes space environment effect.

\section{Experimental scheme of carbon nanotubes atomic oxygen}

In the low earth orbit, space atomic oxygen is one of the primary contributors to material damage $^{[9]}$. The atomic oxygen could denudate, age and contaminate the materials on the surface of spacecrafts, and the denudation extent is closely bound up with the density and distribution change of kinetic energy of atomic oxygen ${ }^{[10-13]}$. The space environment of the low earth orbit is mainly consisting of ultraviolet irradiation and atomic oxygen. The former has greater destruction to organic materials, while the latter is the main destroyer to carbon nanotubes. The experimental technology roadmap of atomic oxygen is as shown in the figure1.

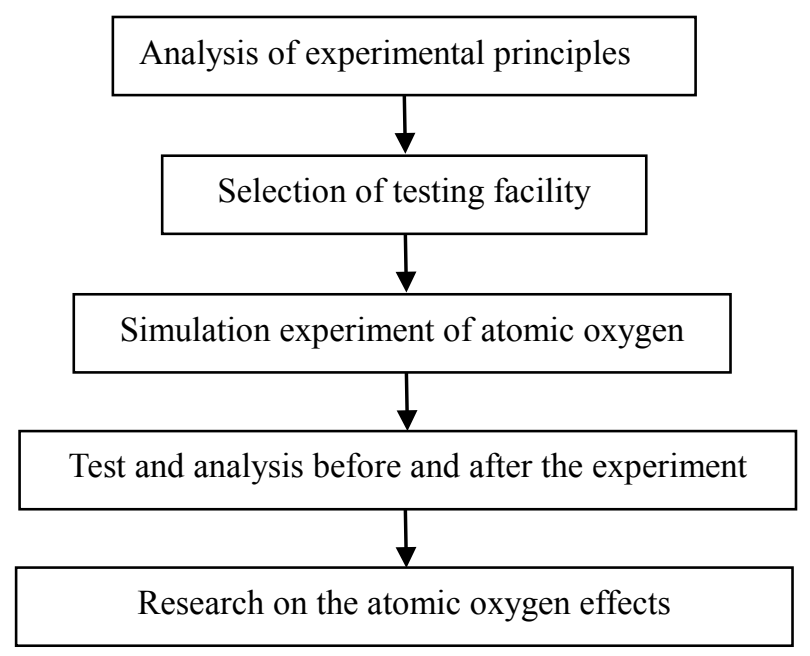

Fig. 1. Experimental technology roadmap of atomic oxygen. 
The microwave ECR ion source and neural plates can be used to simulate the atomic oxygen beam environment. The beam density is calibrated by Kapton $\mathrm{H}$ membrane mass loss method and the exposure time is calculated by the beam density and the beam fluence. The experimental scheme of atomic oxygen requires the carbon nanotubes samples to be put in the testing facility for experiment. The atomic oxygen effect is to be analyzed through the experimental results, appearance, molecular vibration and other information of carbon nanotubes, to ascertain the damage mechanism.

SEM can be employed to determine the carbon nanotubes's atomic oxygen denudation rate, while RAMAN can be applied to measure the carbon nanotubes spectrum. Changes in G zone and $\mathrm{D}$ zone after influenced by grapheme's atomic oxygen is helpful to find out the vibrating model. All this contributes to studying the crystalline defects in the carbon nanotubes and deducing the mechanism through which atomic oxygen damages the carbon nanotubes .

\section{Experimental scheme of the carbon nanotubes's space debris}

The earth orbit space abounds in debris, which has a grave effect on the spacecraft by ultra-high-speed clashes. Space debris has been recognized as one of the environmental factors threatening spacecraft, a manned space station in particular ${ }^{[14,15]}$. When space debris is under test, the carbon nanotubes are different in the number of layers and income shot is distinct in shot size, incident velocity, and incident angle. So, it necessitates studying the damage that carbon nanotubes suffers from hypervelocity impact from multiple perspectives. The experimental technology roadmap of space debris is as shown in the figure 2.

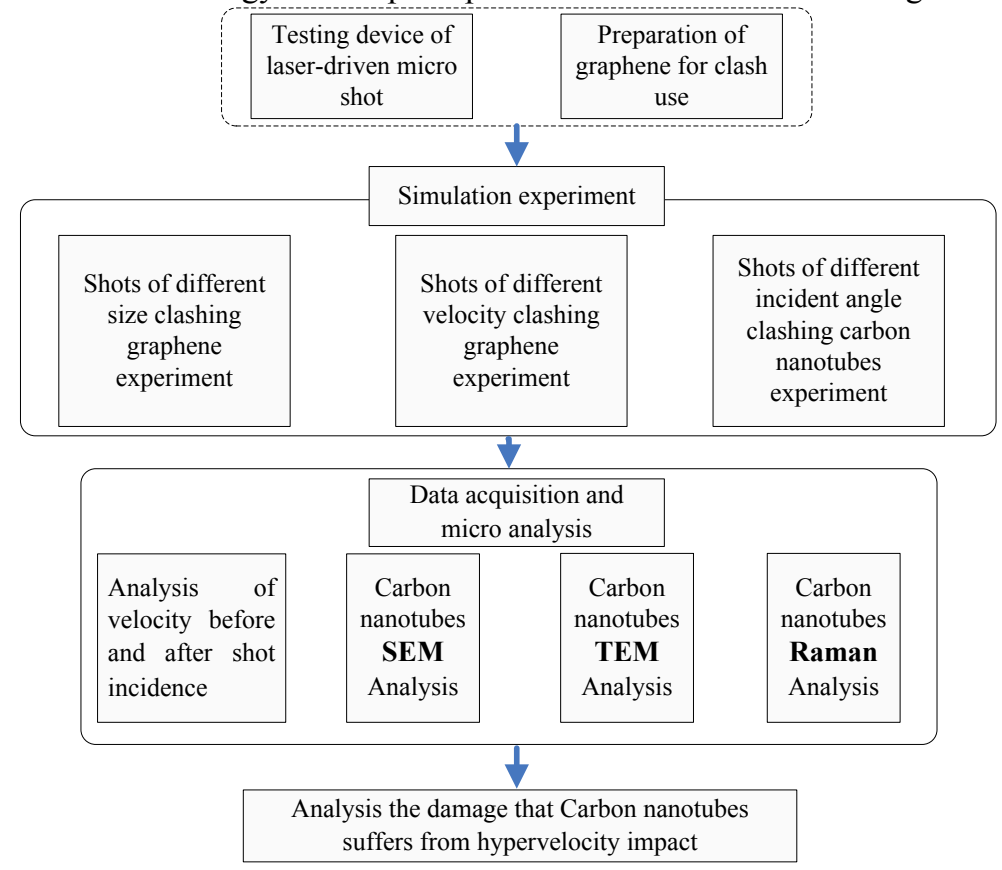

Fig. 2. Experimental technology roadmap of space debris.

The micron-level shot driving technology is primarily represented by plasma driving technology and electrostatic acceleration technology. Compared to these technologies, the laser-driven micro shot technology is simple and easy to operate, free of chemical pollution and electromagnetic interference, allowing controlling the speed of shooting single shot at a single time. The shot is corresponding with the place of clash. 
In terms of regulating the hypervelocity impact, the incidence angle depends on the location where the sample is arranged. The experiment is conducted in a single clash with a single test performed on each sample, thus obtaining an effective data point. The main role of data acquisition and micro analysis is gathering the key data during and after the clash.

\section{Conclusion}

1) Owning to its unique performance, the carbon nanotubes are expected to be widely applied in the spacecraft. It is also affected by the space environment as other aerospace materials. For that reason, it is necessary to perform research on environmental effect including atomic oxygen, high-energy charged particles and space debris. When the environmental effect of carbon nanotubes is determined, it will provide design references to developing composite protective materials.

2) Based on the characteristics of low earth orbit, it is found the main destroyer to carbon nanotubes is atomic oxygen. Therefore, atomic oxygen is a factor that must be taken into account when engaging in the test of simulating the ground environment. The main technological areas that the experiment of atomic oxygen's environmental effect should consider are beam environment simulation, fluence methodology of atomic oxygen as well as the denudation rate of carbon nanotubes.

3) When space debris is under test, it necessitates studying the damage that carbon nanotubes suffers from hypervelocity impact from multiple perspectives. Meanwhile, it is found from the space debris experiment the deformation behavior of carbon nanotubes when stricken at super speed, revealing the mechanism of damage.

4) To explore the behavior characteristics of carbon nanotubes in the space environment and uncover the physical essence that the complicate space environment impacts the micro-nano materials could discover new phenomena, new laws, and new mechanisms, thereby laying a foundation for the development of the discipline-space environmental effects of nanometer materials.

Li Man was funded by the National Natural Science Foundation of China under Grant No.51902026

\section{References}

1. Iskanderova Z A, Kleiman J, Morison W D, et a1. Erosion resistance and durability improvement of polymers and composites in space environment by ion implantation[J]. Materials Chemistry and Physics, 54(1-3): 91-97(1998)

2. Kent B J, Swinyard B M, Maier H J, et al. Boron carbide as atomic oxygen protection for the Lexan-carbon filter on the ROSAT wide-field camera[J]. Multilayer and Grazing Incidence X-Raff EUV Optics, 1546: 312-321(1991)

3. Chen Rongming, Zhang Lei, Yan Chuanwei, et al. Syergic Effect of Atomic Oxygen and Vacuum Ultraviolet on Organic Coatings Degradation[J]. Journal of Aeronautical Materials, 27(1): 41(2007)

4. Wilson J W, Clowdsley M S, Cucinotta F A, et al. Deep space environments for human exploration[J]. Adv Space Res, 34:1281-1287(2004)

5. Wilson J W, Nealy J E, De Angelis G, et al. Deep space environment and shielding[J]. AIP,654:993-1010(2003)

6. Atwell W. Radiation environments for deep-space missions and exposure estimates, AIAA2007-6044[R] 
7. Blakely E A, Chang P Y.A review of ground-based heavy-ion radiobiology relevant to space radiation risk assessment . Part II :Cardiovascular and immunological effects[J].Adv Space Res, 40: 461-469(2007)

8. Atwell W, Reddell B, Boeder P. A comparison of the radiation environments in deep space[J]. SAE 2007 transactions Journal of aerospace, 116(1): 3114(2007)

9. Christian Poivey. Radiation Hardness Assurance for Space System SGT-Inc. NASA GSFC, (2002)

10. Dr.Gary Pippin. Summary Status of MISSE-1 and MISSE-2 Experiments and Details of Estimated Environmental Exposures for and MISSE-2. AFRL- ML- WP- TR- 20064237.

11. Tong Jingyu, Liu Xiangpeng, Zhang Chao, et al. The space atomic oxygen environment erosion on spacecraft surfaces and its protection techniques[J]. Spacecraft Environment Engineering, 26(1): 1-4(2009)

12. Chen Shaohua, Zhang Jiaxun, Yang Sujun, et al. Research on influence of atomic oxygen on organicthermal coat used in spacecraft[J]. Aerospace Materials \& Technology, (4): 33-36(2005)

13. Li Tao. Simulation of space atomic oxygen environment[J]. Spacecraft Environment Engineering, 25(2):134-137(2008)

14. Bernhard R P, Christiansen E L, Kerr J H. Space shuttle meteoroid and orbital debris impact damage . International Journal of Impact Engineering, 25:33-38(2001)

15. Yuan Qingzhi, Sun Yueqiang, Wang Shijin, et al. Research of Micro-Space-Debris Detection[J]. Chinese Journal of Space Science, 25(3)212-217(2005) 